\title{
Algoritmos GRASP e VNS para o Problema de Agendamento de Cirurgias Eletivas em Hospitais de Grande Porte
}

\section{GRASP and VNS algorithms for the Problem of Scheduling of Elective Surgeries in Large Hospitals}

Giselle Paranhos de Andrade ${ }^{1}$, Sérgio Ricardo de Souza², Adriano C. Machado Pereira $^{3}$

\section{RESUMO}

Este artigo trata o Problema de Programação de Cirurgia Eletiva (PACE). O PACE será tratado como um Problema de Programação em Máquinas Paralelas Idênticas, no qual o objetivo é minimizar o tempo de conclusão da última cirurgia. Considera-se neste trabalho o período de agendamento de cirurgias como semanal. Há 5 tipos de movimentos, com base em alocações e trocas, para explorar o espaço de soluções. Os algoritmos desenvolvidos com base em GRASP e VNS foram testados usando 75 instâncias com informações reais de hospitais de Minas Gerais, Brasil. Ao final, foi realizado um teste estatístico t-student para comprovar, com $95 \%$ de confiança a superioridade do algoritmo VNS e sua capacidade em resolver este tipo de problema para as instâncias testadas.

Palavras-chave: Agendamento de cirurgias. Máquinas paralelas. Otimização. Metaheurísticas.

\section{ABSTRACT}

This article deals with the Elective Surgery Programming Problem (PPSE). The PPSE will be treated as a Programming Problem in Identical Parallel Machines, in which the goal is to minimize the time of completion of the last surgery. In this work, it is considered the weekly surgery scheduling period. There are 5 types of movements, based on allocations and exchanges, to explore the space of solutions. The algorithms developed based on GRASP and VNS were tested using 75 instances with real information from hospitals in Minas Gerais, Brazil. At the end, the t-student statistical test was carried out to verify, with $95 \%$ confidence, the superiority of the VNS algorithm and its ability to solve this type of problem for the tested instances.

Keywords: Scheduling of surgeries. Parallel machines. Optimization. Metaheuristics.
${ }^{1}$ Doutoranda em Modelagem Matemática e Computacional, CEFET-MG

E-mail: giselle.acao@gmail.com

${ }^{2}$ Doutorado em Engenharia Elétrica, UNICAMP.

${ }^{3}$ Doutorado, em Ciência da Computação, UFMG. 


\section{INTRODUÇAOO}

O gerenciamento da prestação de serviços de saúde nos hospitais está se tornando cada vez mais importante. Uma unidade de particular interesse é o Centro Cirúrgico (CC), ou melhor, a utilização das salas cirúrgicas, uma vez que o CC é a unidade responsável pelo maior custo do hospital e, segundo Macario et al. (1995), é o principal centro de receita da instituição.

Esta questão é tratada no Problema de Agendamento de Salas Cirúrgicas - PASC (Surgical Case Scheduling - SCS), problema que consiste em alocar recursos hospitalares para casos cirúrgicos e que também define o melhor instante de realização das cirurgias. Este problema tem um papel decisivo na utilização de recursos hospitalares de forma eficiente, segundo Carter e Tovey (1992). O Problema de Agendamento de Salas Cirúrgicas é considerado, na literatura, um problema clássico de otimização combinatória, pertencente à classe NP-Difícil, segundo Carter e Tovey (1992). Logo, as técnicas heurísticas e as metaheurísticas, de maneira geral, têm sido largamente utilizadas na resolução de problemas desta natureza.

Este artigo se concentra em abordar uma versão do PASC denominada PACE (Problema de Agendamento de Cirurgias Eletivas), que consiste em agendar cirurgias previamente conhecidas, desconsiderando casos de cirurgias de emergência. Para a resolução do PACE, é programado um sequenciamento de cirurgias, estabelecendo uma agenda cirúrgica, com o objetivo de minimizar o makespan, ou seja, minimizar o horário de término da última cirurgia, considerando que o período de agendamento tratado é semanal.

O restante deste trabalho está organizado da seguinte forma. Na seção 1.1, é apresentada uma breve revisão bibliográfica para o problema. Na seção 1.2, desenvolvese a caracterização e definição do problema. Na seção 2, apresenta-se a metodologia adotada para a estrutura de dados utilizada. Na seção 2.1, os algoritmos GRASP e VNS são detalhados. Na seção 2.2, são apresentadas as características das instâncias testadas, bem como alguns exemplos. Na seção 3, são expostas as características computacionais para testar as instâncias do problema. Na seção 4, apresenta-se o teste estatístico realizado para validar os resultados obtidos. Por fim, na seção 5, apresenta-se a conclusão do trabalho e a proposta de alguns trabalhos futuros. 


\subsection{Revisão Bibliográfica}

Segundo Ines (2010), o processo de planejamento de cirurgias eletivas pode ser dividido em três fases: Planejamento de Casos Mistos (Case Mix Planning); Planejamento Mestre de Cirurgias (Master Surgery Planning); e Agendamento de Casos Eletivos (Elective Case Scheduling). A fase de Planejamento de Casos Mistos (Case Mix Planning) analisa a disponibilidade, em horas das salas cirúrgicas, distribuída pelos diferentes cirurgiões ou equipes cirúrgicas. Está situada em um nível estratégico de decisão e, geralmente, é realizada anualmente. A distribuição do tempo considera a capacidade operativa de cada cirurgião ou de cada grupo cirúrgico e a quantidade esperada de pacientes ao longo do correspondente horizonte temporal. Hughes e Soliman (1978), Robbins e Tuntiwongpiboon (1989) e Blake e Carter (2002) apresentam diferentes abordagens para esta fase do planejamento. A fase de Planejamento Mestre de Cirurgias (Master Surgery Planning) envolve o desenvolvimento de uma agenda cirúrgica. Trata-se de um documento cíclico, que define o número e o tipo de salas de operações disponíveis, as horas em que as salas estão abertas, definindo, ainda, cirurgiões ou grupos de cirurgias que têm prioridade sobre o tempo das salas cirúrgicas. Esta fase enquadra-se em um nível tático da gestão hospitalar. O horizonte temporal nesta fase do planejamento é mais reduzido do que na primeira fase. Blake e Carter (2002), Blake e Joan (2002) e Belien e Demeulemeester (2007) propõem uma série de modelos para a construção de agendamentos de cirurgias para esta fase. Na fase de Agendamento de Casos Eletivos (Elective Case Scheduling) é estabelecido o agendamento de cada cirurgia em uma base diária. Esta fase situa-se em um nível operacional. Trabalhos relativos a esta fase são encontrados em Magerlein e Martin (1978), Przasnyski (1986), Cardoen et al. (2010), Ozkarahan (1995) e Kharrajal et al. (2006), dentre outros.

Pham e Klinkert (2008) apresentam um modelo em otimização linear inteira mista, baseado em uma extensão do Job Shop Scheduling Problem, denominada Multi-Mode Blocking Job Shop. O modelo define um período de início para cada uma das três fases necessárias na realização de uma cirurgia (pré-operatório, operatório e pós-operatório) e aloca, para cada uma das três fases, um conjunto de recursos necessários. Com técnicas de bloqueio, os autores apresentam uma solução para o problema de restrições de disponibilidade dos equipamentos. É possível, através do algoritmo apresentado, bloquear os recursos indisponíveis no momento da cirurgia. O objetivo é minimizar o período de 
início da última cirurgia a ser realizada. O problema proposto é resolvido com o programa CPLEX.

\subsection{Caracterização do Problema de Agendamento de Cirurgias Eletivas - PACE}

Nesta seção é apresentada uma descrição do Problema de Agendamento de Cirurgias Eletivas (PACE), tratado no presente artigo como um Problema de Programação em Máquinas Paralelas Idênticas (Identical Parallel Machine Scheduling Problem), ou seja, um caso particular de problemas de sequenciamento (Scheduling Problem).

O Problema de Programação em Máquinas Paralelas Idênticas caracteriza-se por um conjunto $N=\{1, \ldots, n\}$ de tarefas, a serem processadas por um conjunto $M=$ $\left\{M_{1}, \ldots, M_{n}\right\}$ de máquinas idênticas, com as seguintes características: (a) cada tarefa deve ser processada exatamente uma vez e por apenas uma máquina; (b) cada tarefa $i$ possui um tempo de processamento $p_{i}$; (c) existem tempos de preparação $s_{i k}$ entre as tarefas $i$ e $k$, considerando que as tarefas $n$ e $k$ serão processadas nesta ordem. Estes tempos de preparação são independentes da sequência, pois é um parâmetro conhecido. O objetivo é encontrar um sequenciamento das $n$ tarefas nas $m$ máquinas de forma a minimizar o tempo de conclusão do sequenciamento, ou seja, o chamado makespan ou $C_{\text {max }}$. Pelas características citadas, este problema é definido como $P \| C_{\max }$, segundo Pinedo (2008).

Com o objetivo de solucionar o PACE usando as características do problema de programação em máquinas paralelas idênticas, considera-se a equivalência entre máquina e sala cirúrgica; e entre tarefa e cirurgia. , Como exemplo, considere, conforme mostra a Tabela 1, os tempos de processamento de sete cirurgias realizadas em duas salas cirúrgicas. A Figura 1 ilustra um possível sequenciamento para este exemplo.

Na Figura 1, observa-se que a cirurgia 6 é alocada na terceira posição da sala $S_{2}$, tendo a cirurgia 4 como predecessora e a cirurgia 3 como sucessora. As partes em preto da figura representam os tempos de preparação ou higienização das salas e equipamentos, entre uma cirurgia e outra. O tempo de conclusão das cirurgias na sala $S_{1}$ é 60 e o da sala $S_{2}$ é 65, o que resulta em um makespan de 65 unidades de tempo.

Tabela 1. Tempos de Processamento das cirurgias

\begin{tabular}{c|ccccccc}
\hline$n$ & 1 & 2 & 3 & 4 & 5 & 6 & 7 \\
\hline \hline$p_{n}$ & 20 & 25 & 15 & 32 & 38 & 23 & 65 \\
\hline
\end{tabular}




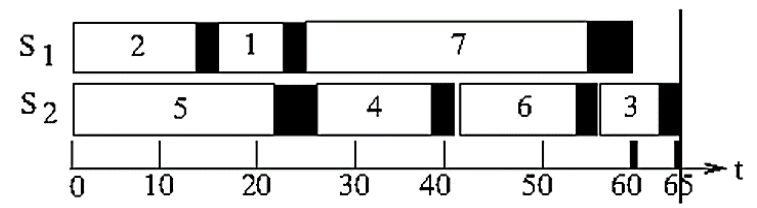

Figura 1. Exemplo de um possível sequenciamento

\section{METODOLOGIA}

Esta seção apresenta os procedimentos propostos para a solução do Problema de Agendamento de Cirurgias Eletivas (PACE). Inicialmente, é mostrada a estrutura de dados utilizada para a representação de uma solução. Em seguida, apresenta-se os cinco tipos de movimentos utilizados na vizinhança da solução, bem como a busca local utilizada como heurística de refinamento. Após, apresenta-se as metaheurísticas implementadas, a saber, Greedy Randomized Adaptive Search Procedure (GRASP) e Variable Neighborhood Search (VNS).

\section{Representação da Solução}

A solução do PACE é representada por dois vetores. O primeiro vetor, intitulado vetorseq, representa a sequência em que as cirurgias devem ser realizadas, ao passo que o segundo vetor, chamado aqui de vetor sala, representa em quais das salas a cirurgia será realizada, visto que cada cirurgia pode ser realizada em mais de uma sala. A Figura 2 representa a solução para o exemplo apresentado na seção anterior. Verifica-se que as cirurgias de número 1, 2 e 7 são realizadas na sala 1 e as demais na sala 2.

\begin{tabular}{c|c|c|c|c|c|c|c|} 
& \multicolumn{1}{c}{1} & 2 & 3 & 4 & 5 & 6 & 7 \\
\cline { 2 - 8 } vetor seq & 2 & 5 & 1 & 4 & 7 & 6 & 3 \\
\cline { 2 - 8 } & 1 & 2 & 3 & 4 & 5 & 6 & 7 \\
\cline { 2 - 8 } vetor sala & 1 & 1 & 2 & 2 & 2 & 2 & 1 \\
\cline { 2 - 8 }
\end{tabular}

Figura 2. Representação da solução através de dois vetores

\section{Vizinhança}

Para exploração do espaço de soluções, foram utilizadas combinações de dois tipos diferentes de movimentos: troca e realocação, conforme descrito a seguir.

Movimento1 - TrocaOrdemCirurgias: consiste em realizar as trocas de posições entre duas cirurgias realizadas em quaisquer salas;

Movimento2 - RealocaSala: consiste em realocar uma cirurgia para uma outra sala;

Movimento3 - TrocaOrdemCirurgias2: consiste em realizar o Movimento1 duas vezes 
no vetor de ordem das cirurgias;

Movimento4 - RealocaSala2: consiste em realizar o Movimento2 duas vezes no vetor de salas;

Movimento5 - TrocaRealoca: consiste em realizar o Movimento1 seguido do movimento2.

\section{Busca Local}

Nesta implementação, é utilizado, como heurística de refinamento, o Método Randômico de Descida. A utilização desse método se justifica pela dimensão do espaço de busca do problema tratado e pelo número de combinações e movimentos possíveis na exploração do mesmo. O método implementado consiste em analisar um conjunto de vizinhos gerados a partir de movimentos aleatórios, em que o melhor vizinho encontrado é comparado com a solução corrente. A solução vizinha somente será a nova solução corrente caso seja melhor que a solução corrente. Para a geração da vizinhança, a cada iteração, todos os movimentos descritos na seção anterior têm a mesma probabilidade de ocorrerem. No Algoritmo 1, é apresentado o pseudocódigo da busca local utilizada.

Algoritmo 1. Busca Local - Método Randômico de Descida

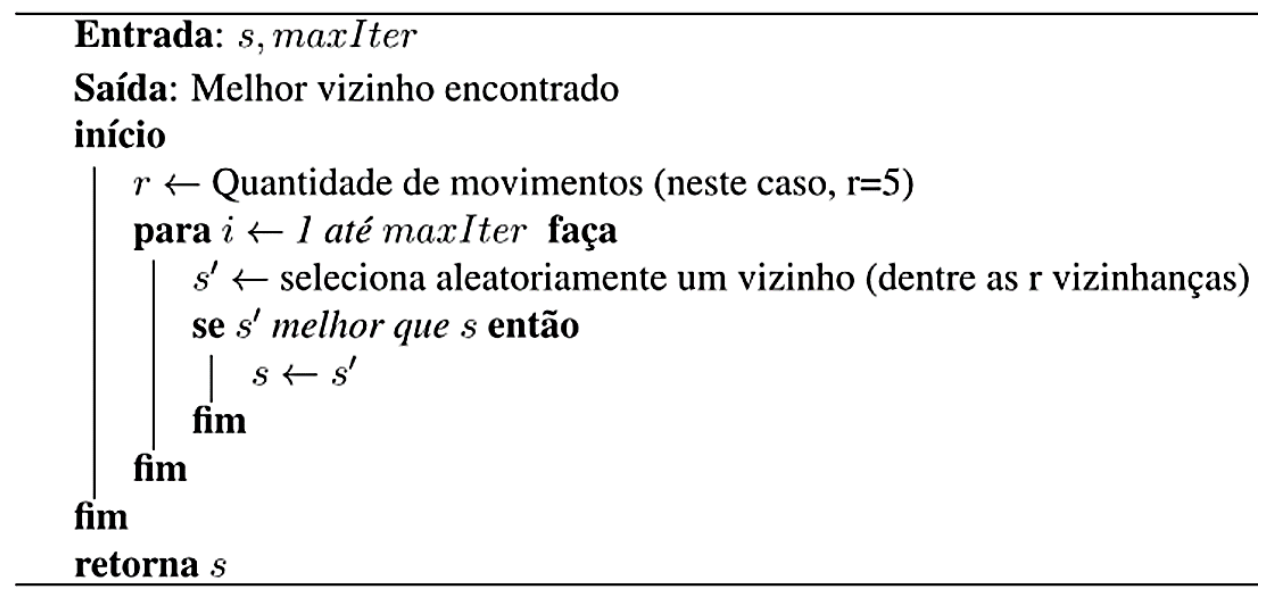

\subsection{Metaheurísticas Implementadas}

\section{Greedy Randomized Adaptive Search Procedure (GRASP)}

A metaheurística Greedy Randomized Adaptive Search Procedure (GRASP), proposta por Feo e Resende (1995), é um processo iterativo no qual cada iteração consiste em duas fases: (i) fase construtiva, que gera soluções factíveis para o problema; e (ii) fase de busca local, que busca o ótimo local na vizinhança das soluções iniciais, geradas pela fase de construção. 
$\mathrm{Na}$ fase construtiva, uma função denominada construçãoGRASP é empregada, gerando uma Lista de Candidatos (LC). Tais candidatos são avaliados segundo um critério em que o tempo necessário para a realização de uma cirurgia seja maior ou igual à diferença entre $T_{\max }$ e $\alpha\left(T_{\max }-T_{\min }\right)$, sendo $\alpha$ um parâmetro real variando entre 0 e 1. Para $\alpha=1$, tem-se uma solução totalmente aleatória; para $\alpha=0$, tem-se uma solução gulosa. Os candidatos que atendem a esta condição compõem a Lista Restrita de Candidatos (LRC). Logo, o algoritmo irá escolher aleatoriamente um dos candidatos da LRC, sequenciando-o e retirando-o da LC. Este passo se repete até que todas as tarefas sejam sequenciadas.

Para a segunda fase ou fase de busca local foi utilizado o Método Randômico de Descida, já descrita na seção anterior. Como já dito anteriormente, este método analisa parte da vizinhança da solução corrente. Os movimentos e vizinhos são escolhidos aleatoriamente. No final, efetua-se uma comparação entre a melhor solução encontrada e a solução corrente. Havendo melhora, a solução corrente é atualizada, senão a solução permanece inalterada.

O pseudocódigo do GRASP é apresentado no Algoritmo 2. Uma solução é construída de forma parcialmente gulosa com o método chamado construçãoGRASP, que consiste em escolher aleatoriamente a próxima cirurgia a entrar no sequenciamento, dentre uma lista de melhores candidatos. Essa lista é montada de acordo com a regra de menores tempos de execução. O parâmetro $\alpha$ define o tamanho da lista. Em seguida é feito um refinamento na solução através de busca local. A melhor solução é atualizada se houver melhora. Esses passos são repetidos até que um critério de parada seja atendido.

\section{Variable Neighborhood Search (VNS)}

A metaheurística Variable Neighborhood Search (VNS), proposta por Mladenovic e Hansen (1997), é uma metaheurística que se baseia em mudanças sistemáticas de vizinhança das soluções para resolver problemas de otimização. O método VNS explora vizinhanças gradativamente mais distantes da solução corrente e focaliza a busca em torno de uma nova solução se e somente se um movimento de melhora é realizado. $\mathrm{O}$ VNS inclui, também, um procedimento de busca local a ser aplicado sobre a solução corrente.

O pseudocódigo do VNS é apresentado no Algoritmo 3. É construída uma solução aleatória. Em seguida, a solução construída é refinada através de busca local, utilizando o método de Descida Aleatória, já explicado anteriormente. Depois um vizinho é escolhido 
aleatoriamente na vizinhança $r$. Após este procedimento, é refinada e, se houver melhora na solução, ela é atualizada e a vizinhança reinicializada.

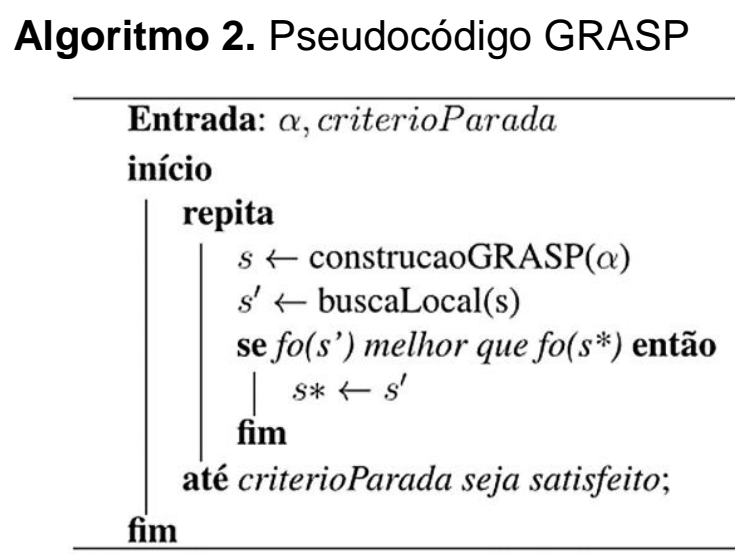

\section{Algoritmo 3. Pseudocódigo VNS}

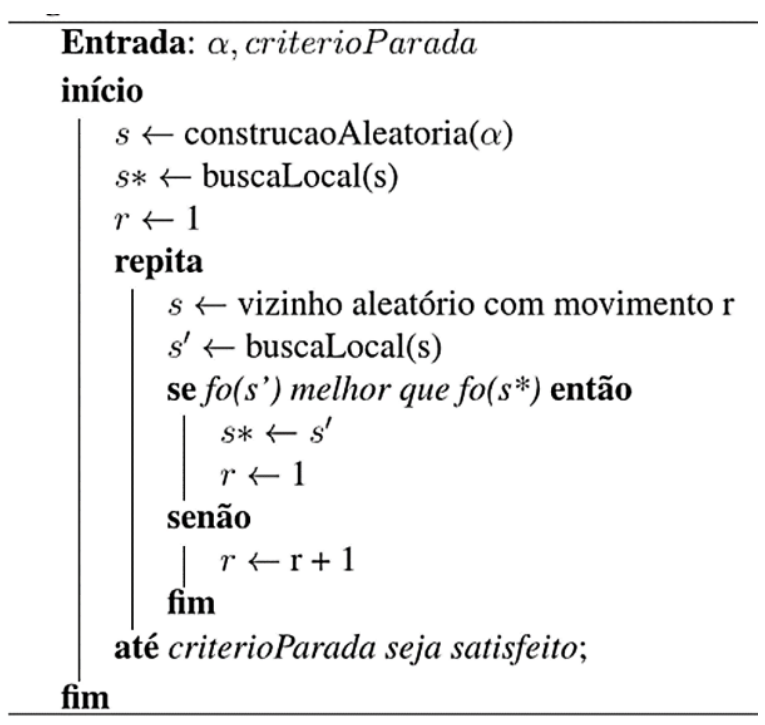

\subsection{Instâncias Propostas para o PACE}

As instâncias utilizadas para efetuar os testes computacionais nos algoritmos desenvolvidos, apresentados na seção 2.1, foram geradas a partir de dados reais obtidos em quatro hospitais de grande porte localizados na região metropolitana de Belo Horizonte, pesquisados no ano de 2017, sendo três hospitais da rede privada e um hospital da rede pública. Na Tabela 2, é apresentado um exemplo de como são esquematizadas as informações contidas nas instâncias geradas e testadas.

Tabela 2. Informações das Instâncias

\begin{tabular}{c|c|c|c|c|c|c|c}
\hline Instância & Cirurgias & $\begin{array}{c}\text { Salas } \\
\text { Cirúrgicas }\end{array}$ & $\begin{array}{c}\text { Salas } \\
\text { de RPA }\end{array}$ & $\begin{array}{c}\text { Leitos } \\
\text { de UTI }\end{array}$ & Cirurgiões & Enfermeiros & Anestesistas \\
\hline
\end{tabular}


ANDRADE, G.P.; SOUZA, S.R.; PEREIRA, A.C.M

Algoritmos GRASP e VNS para o Problema de Agendamento de Cirurgias Eletivas em Hospitais de Grande Porte.

Tabela 3. Instâncias Testadas

\begin{tabular}{|c|c|c|c|c|c|c|c|}
\hline Instância & Cirurgias & $\begin{array}{c}\text { Salas } \\
\text { Cirúrgicas }\end{array}$ & $\begin{array}{c}\text { Salas } \\
\text { de RPA }\end{array}$ & $\begin{array}{l}\text { Leitos } \\
\text { de UTI }\end{array}$ & Cirurgiões & Enfermeiros & Anestesistas \\
\hline h1 & 162 & 7 & 6 & 24 & 74 & 18 & 20 \\
\hline h2 & 192 & 12 & 15 & 29 & 112 & 21 & 25 \\
\hline h3 & 216 & 18 & 20 & 40 & 126 & 27 & 31 \\
\hline h4 & 266 & 16 & 55 & 18 & 157 & 34 & 37 \\
\hline h5 & 354 & 19 & 21 & 53 & 186 & 39 & 45 \\
\hline h6 & 378 & 25 & 26 & 64 & 200 & 45 & 51 \\
\hline h7 & 428 & 23 & 61 & 42 & 231 & 52 & 57 \\
\hline h8 & 408 & 30 & 35 & 69 & 238 & 48 & 56 \\
\hline h9 & 458 & 28 & 70 & 47 & 269 & 55 & 62 \\
\hline h10 & 482 & 34 & 75 & 58 & 283 & 61 & 68 \\
\hline h11 & 570 & 37 & 41 & 93 & 312 & 66 & 76 \\
\hline h12 & 620 & 35 & 76 & 71 & 343 & 73 & 82 \\
\hline h13 & 644 & 41 & 81 & 82 & 357 & 79 & 88 \\
\hline h14 & 674 & 46 & 90 & 87 & 395 & 82 & 93 \\
\hline h15 & 836 & 53 & 96 & 111 & 469 & 100 & 113 \\
\hline h16 & 162 & 6 & 6 & 24 & 74 & 18 & 20 \\
\hline h17 & 192 & 11 & 15 & 29 & 112 & 21 & 25 \\
\hline h18 & 216 & 16 & 20 & 40 & 126 & 27 & 31 \\
\hline h19 & 266 & 14 & 55 & 18 & 157 & 34 & 37 \\
\hline h20 & 354 & 17 & 21 & 53 & 186 & 39 & 45 \\
\hline h21 & 378 & 22 & 26 & 64 & 200 & 45 & 51 \\
\hline h22 & 428 & 20 & 61 & 42 & 231 & 52 & 57 \\
\hline h23 & 408 & 27 & 35 & 69 & 238 & 48 & 56 \\
\hline h24 & 458 & 25 & 70 & 47 & 269 & 55 & 62 \\
\hline h25 & 482 & 30 & 75 & 58 & 283 & 61 & 68 \\
\hline h26 & 570 & 33 & 41 & 93 & 312 & 66 & 76 \\
\hline h27 & 620 & 31 & 76 & 71 & 343 & 73 & 82 \\
\hline h28 & 644 & 36 & 81 & 82 & 357 & 79 & 88 \\
\hline h29 & 674 & 41 & 90 & 87 & 395 & 82 & 93 \\
\hline h30 & 836 & 47 & 96 & 111 & 469 & 100 & 113 \\
\hline h31 & 162 & 7 & 6 & 24 & 67 & 18 & 20 \\
\hline h32 & 192 & 12 & 15 & 29 & 100 & 21 & 25 \\
\hline h33 & 216 & 18 & 20 & 40 & 114 & 27 & 31 \\
\hline h34 & 266 & 16 & 55 & 18 & 140 & 34 & 37 \\
\hline h35 & 354 & 19 & 21 & 53 & 167 & 39 & 45 \\
\hline h36 & 378 & 25 & 26 & 64 & 181 & 45 & 51 \\
\hline h37 & 428 & 23 & 61 & 42 & 207 & 52 & 57 \\
\hline h38 & 408 & 30 & 35 & 69 & 214 & 48 & 56 \\
\hline h39 & 458 & 28 & 70 & 47 & 240 & 55 & 62 \\
\hline h40 & 482 & 34 & 75 & 58 & 254 & 61 & 68 \\
\hline h41 & 570 & 37 & 41 & 93 & 281 & 66 & 76 \\
\hline h42 & 620 & 35 & 76 & 71 & 307 & 73 & 82 \\
\hline h43 & 644 & 41 & 81 & 82 & 321 & 79 & 88 \\
\hline h44 & 674 & 46 & 90 & 87 & 354 & 82 & 93 \\
\hline h45 & 836 & 53 & 96 & 111 & 421 & 100 & 113 \\
\hline h46 & 162 & 8 & 6 & 24 & 74 & 18 & 20 \\
\hline h47 & 192 & 13 & 15 & 29 & 112 & 21 & 25 \\
\hline h48 & 216 & 20 & 20 & 40 & 126 & 27 & 31 \\
\hline h49 & 266 & 18 & 55 & 18 & 157 & 34 & 37 \\
\hline h50 & 354 & 21 & 21 & 53 & 186 & 39 & 45 \\
\hline h51 & 378 & 28 & 26 & 64 & 200 & 45 & 51 \\
\hline h52 & 428 & 26 & 61 & 42 & 231 & 52 & 57 \\
\hline h53 & 408 & 33 & 35 & 69 & 238 & 48 & 56 \\
\hline h54 & 458 & 31 & 70 & 47 & 269 & 55 & 62 \\
\hline h55 & 482 & 38 & 75 & 58 & 283 & 61 & 68 \\
\hline h56 & 570 & 41 & 41 & 93 & 312 & 66 & 76 \\
\hline h57 & 620 & 39 & 76 & 71 & 343 & 73 & 82 \\
\hline h58 & 644 & 46 & 81 & 82 & 357 & 79 & 88 \\
\hline h59 & 674 & 51 & 90 & 87 & 395 & 82 & 93 \\
\hline h60 & 836 & 59 & 96 & 111 & 469 & 100 & 113 \\
\hline h61 & 162 & 7 & 6 & 24 & 81 & 18 & 20 \\
\hline h62 & 192 & 12 & 15 & 29 & 124 & 21 & 25 \\
\hline h63 & 216 & 18 & 20 & 40 & 138 & 27 & 31 \\
\hline h64 & 266 & 16 & 55 & 18 & 174 & 34 & 37 \\
\hline h65 & 354 & 19 & 21 & 53 & 205 & 39 & 45 \\
\hline h66 & 378 & 25 & 26 & 64 & 219 & 45 & 51 \\
\hline h67 & 428 & 23 & 61 & 42 & 255 & 52 & 57 \\
\hline h68 & 408 & 30 & 35 & 69 & 262 & 48 & 56 \\
\hline h69 & 458 & 28 & 70 & 47 & 298 & 55 & 62 \\
\hline h70 & 482 & 34 & 75 & 58 & 312 & 61 & 68 \\
\hline h71 & 570 & 37 & 41 & 93 & 343 & 66 & 76 \\
\hline h72 & 620 & 35 & 76 & 71 & 379 & 73 & 82 \\
\hline h73 & 644 & 41 & 81 & 82 & 393 & 79 & 88 \\
\hline h74 & 674 & 46 & 90 & 87 & 436 & 82 & 93 \\
\hline h75 & 836 & 53 & 96 & 111 & 517 & 100 & 113 \\
\hline
\end{tabular}


Na Tabela 2, tem-se que:

- Instância: representa a instância, denotada pelo prefixo $h$;

- Cirurgias: quantidade de cirurgias previamente conhecida para o agendamento semanal;

- Salas Cirúrgicas: quantidade de salas cirúrgicas disponíveis;

- Salas de RPA: quantidade de salas de recuperação pós-anestésica disponíveis;

- Leitos de UTI: quantidade de leitos de UTI disponíveis;

- Cirurgiões: quantidade de cirurgiões disponíveis;

- Enfermeiros: quantidade de enfermeiros disponíveis;

- Anestesistas: quantidade de anestesistas disponíveis.

Os detalhes de cada instância podem ser encontrados na Tabela 3.

\section{RESULTADOS}

Os algoritmos GRASP e VNS foram implementados em linguagem $\mathrm{C}++$. As instâncias têm os tempos de cirurgias gerados aleatoriamente entre 2 e 16 slots de tempo, com distribuição binomial mais um fator constante 1, se o tempo for menor que 8, e 2, caso contrário.

Os testes computacionais foram realizados em um computador com processador Intel Core i3-M330, 2,13 GHz, 3GB de RAM e sistema operacional Ubuntu 16.04 de 32 bits usando compilador $\mathrm{G}_{++}$versão 5.4. Foram realizadas 30 execuções de cada algoritmo sobre cada instância, tendo-se como critério de parada o número de iterações, no caso, 100 iterações. Na Tabela 4 são apresentados os resultados obtidos, sendo estes melhores valores de avaliação encontrados (Média (GRASP - VNS)), (Desvio Padrão), (teste-t (H_1:f_VNS<f_GRASP; 95\%)), (Quantidade), onde consta a superioridade do algoritmo VNS, o qual reduz o valor de makespan em 66 das 75 instâncias testadas.

\section{DISCUSSAOO}

Para análise estatística e comprovação da existência de diferenças significativas entre os algoritmos implementados, foram utilizados vários testes $t$-Student de uma amostra unicaudal à esquerda. Considerando-se $\mu_{0}=\{0,8,10,12\}$ e $H_{1}: \mu>\mu_{0}$. Seja $f_{A} 0$ valor de função objetivo alcançado pelo algoritmo $A$ e $t_{A}$ o tempo de execução do algoritmo $A$. Seguem os seguintes modelos dos testes de hipóteses:

$$
\left\{\begin{array} { l } 
{ H _ { 1 } : t _ { G R A S P } < t _ { V N S } } \\
{ H _ { 0 } : t _ { G R A S P } \geq t _ { V N S } }
\end{array} \quad \left\{\begin{array}{l}
H_{1}: f_{G R A S P}-f_{V N S}>\alpha \\
H_{0}: f_{G R A S P}-f_{V N S} \leq \alpha
\end{array}, \forall \alpha \in\{0,8,10,12\}\right.\right.
$$

Após analisar 30 execuções de cada algoritmo, em cada uma das 75 instâncias, conforme resultado na Tabela 4, percebe-se que:

- É possível afirmar com significância de 95\% que a resposta do algoritmo VNS tem um valor de função objetivo menor que as do algoritmo GRASP para 66 das 75 instâncias testadas, lembrando que o problema é de minimização.

- Com 95\% de confiança, o algoritmo VNS reduz o makespan em mais de 8 slots de tempo em 29 das 66 instâncias onde houve melhora.

- Com 95\% de confiança, o algoritmo VNS reduz o makespan em mais de 10 slots de tempo em 22 das 66 instâncias onde houve melhora.

- Com 95\% de confiança, o algoritmo VNS reduz o makespan em mais de 12 slots de tempo em 8 das 66 instâncias onde houve melhora. 
ANDRADE, G.P.; SOUZA, S.R.; PEREIRA, A.C.M

Algoritmos GRASP e VNS para o Problema de Agendamento de Cirurgias Eletivas em Hospitais de Grande Porte.

- Com relação ao tempo de execução, pode-se afirmar com mais de $99 \%$ de confiança que o algoritmo GRASP é cerca de 3,5 vezes mais rápido que o VNS em todos os casos.

Tabela 4. Resultados

\begin{tabular}{|c|c|c|c|c|}
\hline & Média (GRASP - VNS) & Desvio Padrão & teste-t $\left(H_{1}: f_{V N S}<f_{G R A S P} ; 95 \%\right)$ & Quantidade \\
\hline h1 & 5,90 & 9,02239 & 0,03029 & 1 \\
\hline h2 & 3,77 & 6,19612 & 0,00308 & 1 \\
\hline h3 & 6,17 & 10,50479 & 0,00121 & 1 \\
\hline h4 & 8,70 & 8,89847 & 0,12160 & 0 \\
\hline h5 & 10,13 & 12,05085 & 0,00992 & 1 \\
\hline h6 & 10,60 & 10,73056 & 0,01345 & 1 \\
\hline h7 & 8,90 & 11,66294 & 0,04773 & 1 \\
\hline h8 & 7,67 & 10,71040 & 0,00045 & 1 \\
\hline h9 & 8,87 & 15,73648 & 0,02517 & 1 \\
\hline h10 & 17,13 & 14,19503 & 0,00018 & 1 \\
\hline h11 & 16,80 & 17,89124 & 0,36330 & 0 \\
\hline h12 & 13,57 & 16,00147 & 0,01947 & 1 \\
\hline h13 & 16,10 & 11,34217 & 0,08686 & 0 \\
\hline h14 & 16,27 & 20,52237 & 0,01994 & 1 \\
\hline h15 & 15,73 & 15,98476 & 0,19173 & 0 \\
\hline h16 & 10,23 & 12,37262 & 0,00145 & 1 \\
\hline h17 & 5,23 & 7,26201 & 0,00001 & 1 \\
\hline h18 & 11,50 & 12,19596 & 0,00855 & 1 \\
\hline h19 & 6,90 & 8,10002 & 0,04556 & 1 \\
\hline h20 & 6,53 & 8,50044 & 0,00433 & 1 \\
\hline h21 & 10,20 & 11,17077 & 0,00124 & 1 \\
\hline h22 & 7,57 & 11,09422 & 0,00147 & 1 \\
\hline h23 & 12,57 & 13,41045 & 0,00506 & 1 \\
\hline h24 & 12,40 & 14,69835 & 0,00003 & 1 \\
\hline h25 & 15,40 & 17,92244 & 0,21713 & 0 \\
\hline h26 & 11,83 & 12,80647 & 0,00017 & 1 \\
\hline h27 & 10,10 & 12,28217 & 0,02025 & 1 \\
\hline h28 & 11,60 & 13,18201 & 0,00001 & 1 \\
\hline h29 & 16,80 & 16,19366 & 0,02605 & 1 \\
\hline h30 & 17,57 & 18,04340 & 0,00079 & 1 \\
\hline h31 & 7,10 & 10,61343 & 0,11177 & 0 \\
\hline h32 & 6,87 & 10,13609 & 0,01812 & 1 \\
\hline h33 & 7,07 & 9,05132 & 0,00879 & 1 \\
\hline h34 & 6,00 & 9,28105 & 0,00006 & 1 \\
\hline h35 & 11,40 & 13,04792 & 0,00067 & 1 \\
\hline h36 & 10,07 & 11,79402 & 0,00386 & 1 \\
\hline h37 & 6,97 & 8,63227 & 0,00044 & 1 \\
\hline h38 & 10,37 & 13,52771 & 0,00046 & 1 \\
\hline h39 & 7,90 & 9,35267 & 0,03299 & 1 \\
\hline h40 & 12,13 & 12,74615 & 0,00159 & 1 \\
\hline h41 & 19,93 & 17,44337 & 0,00359 & 1 \\
\hline h42 & 16,63 & 15,85165 & 0,00416 & 1 \\
\hline h43 & 16,00 & 18,90265 & 0,00094 & 1 \\
\hline h44 & 21,00 & 18,13551 & 0,03129 & 1 \\
\hline h45 & 9,10 & 12,11539 & 0,00245 & 1 \\
\hline h46 & 4,83 & 7,25441 & 0,02660 & 1 \\
\hline h47 & 5,50 & 7,17154 & 0,00277 & 1 \\
\hline h48 & 9,17 & 10,51135 & 0,01629 & 1 \\
\hline h49 & 7,03 & 9,41196 & 0,00576 & 1 \\
\hline h50 & 9,93 & 10,32217 & 0,00462 & 1 \\
\hline h51 & 10,00 & 12,41523 & 0,01028 & 1 \\
\hline h52 & 4,73 & 7,93914 & 0,00003 & 1 \\
\hline h53 & 8,63 & 8,89976 & 0,00277 & 1 \\
\hline h54 & 13,50 & 13,54367 & 0,18564 & 0 \\
\hline h55 & 14,63 & 13,28853 & 0,05643 & 0 \\
\hline h56 & 16,63 & 16,78358 & 0,00922 & 1 \\
\hline h57 & 12,53 & 15,45345 & 0,00014 & 1 \\
\hline h58 & 11,43 & 13,13244 & 0,00062 & 1 \\
\hline h59 & 14,57 & 14,75240 & 0,00683 & 1 \\
\hline h60 & 25,30 & 18,37755 & 0,02270 & 1 \\
\hline h61 & 6,37 & 9,65074 & 0,00614 & 1 \\
\hline h62 & 6,47 & 10,44108 & 0,02758 & 1 \\
\hline h63 & 8,07 & 9,78364 & 0,13333 & 0 \\
\hline h64 & 6,60 & 8,52420 & 0,00044 & 1 \\
\hline h65 & 6,07 & 7,58371 & 0,00185 & 1 \\
\hline h66 & 11,97 & 12,15839 & 0,00301 & 1 \\
\hline h67 & 15,60 & 19,00744 & 0,00876 & 1 \\
\hline h68 & 11,10 & 12,39953 & 0,01894 & 1 \\
\hline h69 & 13,27 & 13,49823 & 0,00004 & 1 \\
\hline h70 & 18,90 & 16,19994 & 0,00478 & 1 \\
\hline h71 & 18,20 & 21,12100 & 0,02872 & 1 \\
\hline h72 & 21,20 & 18,27076 & 0,00166 & 1 \\
\hline h73 & 15,60 & 18,57993 & 0,00193 & 1 \\
\hline h74 & 15,03 & 14,49015 & 0,00282 & 1 \\
\hline h75 & 14,43 & 14,63271 & 0,01231 & 1 \\
\hline
\end{tabular}




\section{CONSIDERAÇOES FINAIS}

Este trabalho apresentou um estudo acerca da aplicação de metaheurísticas para resolução do Problema de Agendamento de Cirurgias Eletivas em Hospitais de Grande Porte - PACE. Abordou-se aqui a metodologia de Programação de Máquinas Paralelas, uma classe de problemas de agendamento, em que se pretende realizar o agendamento semanal de cirurgias previamente conhecidas, com o objetivo de minimizar o makespan, ou instante de término da última cirurgia. São considerados, neste trabalho, a disponibilidade dos recursos de sala cirúrgica, sala de recuperação pós-anestésica (RPA), leitos de UTI, cirurgiões, anestesistas e enfermeiros. Para solucionar o PACE, foram implementadas as metaheurísticas GRASP e VNS, a busca local ou heurística de refinamento utilizada foi o Método Randômico de Descida. A utilização desta busca local se justifica pelo grande espaço de busca que o problema apresenta. Por meio deste trabalho, foi possível testar e desenvolver diferentes técnicas, de forma adaptada ao PACE, permitindo a obtenção de soluções de maior qualidade, quando comparadas com as soluções encontradas atualmente nos hospitais. Com a finalidade de testar a metodologia proposta, foram geradas instâncias com dados reais de quatro hospitais de grande porte pesquisados durante o ano de 2017. Foram geradas 75 instâncias, contendo até 836 cirurgias semanais, a serem agendadas em 53 salas cirúrgicas. De maneira geral, utilizando as instâncias geradas, os algoritmos desenvolvidos apresentaram resultados muito promissores, sendo capazes de encontrar soluções melhores do que as soluções presentes, atualmente, nos hospitais em 100\% das instâncias testadas, como é o caso da metaheurística VNS que se destaca para este grupo de instâncias.

Conclui-se com este trabalho e com os resultados apresentados que o algoritmo VNS contribui para a resolução do Problema de Agendamento de Cirurgias Eletivas. Demonstrando assim a grande importância de explorar várias vizinhanças e manter movimentos mais robustos.

Para análise estatística e comprovação da existência de diferenças significativas entre os algoritmos implementados, foram utilizados vários testes $t$-Student de uma amostra unicaudal à esquerda.

Como proposta de trabalhos futuros, os autores consideram:

-Testar e propor um agendamento mensal para o PACE;

-Testar um método exato, usando Optimization Programming Language (OPL);

- Implementar a inclusão de agendamento de cirurgias de emergência a atual agenda;

-Implementar com a linguagem de programação Python usando bibliotecas de heurísticas.

\section{REFERENCIAS}

AIEX, R.M.; RESENDE, M.G.C.; RIBEIRO, C.C. Probability distribution of solution time in grasp: an experimental investigation. Journal of Heuristics, v. 8, 343-373, 2002. 
BELIEN, J.; DEMEULEMEESTER, E. Building cyclic master surgery schedules with leveled resulting bed occupancy. European Journal of Operational Research, v. 2, n. 176, 1185-1204, 2007.

BLAKE, J.T.; CARTER, M.W. A goal programming approach to strategic resource allocation in acute care hospitals. European Journal of Operational Research, v. 140, 541-561, 2002.

BLAKE, J.T.; JOAN, D. Mount sinai hospital uses integer programming to allocate operating room time. Interfaces, v. 32, n. 2, 63-73, 2002.

CARDOEN, B.; DEMEULEMEESTER, E.; BELIËN, J. Operating room planning and scheduling: a literature review. European Journal of Operational Research, v. 3, 333333,2010 .

CARTER, M.W.; TOVEY, C.A. When is the classroom assignment problem hard? Operations Research, v. 40, n. 1, 28-30, 1992.

DEXTER, F.; TRAUB, R.D. How to schedule elective surgical cases into specific operating rooms to maximize the efficiency of use of operating room time. Anesthesia and Analgesia, v. 94, n. 4, 933-942, 2002.

FEO, T.A.; RESENDE, M.G.C. Greedy randomized adaptive search procedures. Journal of Global Optimization, 109-133, 1995.

FIELD, A.P. Descobrindo a estatística usando o SPSS. 2 edição, 2009.

HUGHES, W.L.; SOLIMAN, S.Y. Short-term case mix management with linear programming. Hospital \& Health Services Administration, v. 30,52-60, 1978.

KHARRAJAL, S.; ALBERT, P.; CHAABANEL, S. Bloco de programação para um calendário cirúrgico. In: CONFERÊNCIA INTERNACIONAL SOBRE SISTEMAS DE SERVIÇOS E SISTEMAS DE GERENCIAMENTO. Anais..., 2006.

LOURENÇO, H.R.; MARTIN, O.C.; STÜTZLE, T. Iterated local search. Handbook of Metaheuristics. Kluwer Academic Publishers, Boston, 321-353, 2003.

MACARIO, A.; VITEZ, T.S.; DUNN, B.; MCDONALD, T. Where are the costs in perioperative care?: analysis of hospital costs and charges for inpatient surgical care. Anesthesiology, v. 83, n. 6, 1138-1144, 1995.

MAGERLEIN, J.M.; MARTIN, J.B. Surgical demand scheduling: a review. Health Services Research, 418-433, 1978.

MLADENOVIC, N; HANSEN, P. Variable neighborhood search. Computers and Operations Research, v. 24, 1097-1110, 1997.

OZKARAHAN, I. Allocation of surgical procedures to operating rooms. Journal of Medical Systems, v. 4, n. 19, 333-352, 1995.

PHAM, D.N.; KLINKERT, A. Surgical case scheduling as a generalized job shop scheduling problem. v. 185,1011-1025, 2008.

PINEDO, M. Scheduling: theory, algorithms, and systems. Springer Verlag, 2008. 
PROENÇA, I.M. Planejamento de cirurgias eletivas: abordagens em programação inteira. Tese de Doutorado, Departamento de Estatística e Investigação Operacional, Universidade de Lisboa, 2010.

PRZASNYSKI, Z.H. Operating room scheduling: a literature review. AORN Journal, v. 44, 67-79, 1986.

ROBBINS, W.A.; TUNTIWONGPIBOON, N. Linear programming is a useful tool in casemix management. Healthcare Financial Management, v. 6, n. 43, 114-116, 1989. 